Chapman University

Chapman University Digital Commons

Mathematics, Physics, and Computer Science Science and Technology Faculty Articles and Faculty Articles and Research

Research

$9-17-2003$

\title{
Refrigerator with Phonon Filters: An Application of the Phonon Deficit Effect in Superconducting Tunnel Junctions
}

\author{
G. G. Melkonyan \\ Université Laval \\ Armen Gulian \\ Chapman University, gulian@chapman.edu
}

Follow this and additional works at: https://digitalcommons.chapman.edu/scs_articles

Part of the Condensed Matter Physics Commons, and the Other Physics Commons

\section{Recommended Citation}

Melkonyan G.G., Kroger H., and Gulian A.M. Refrigerator with phonon filters: An application of the phonon deficit effect in superconducting tunnel junctions. J. Appl. Phys., 2003, vol. 94, No. 7, pp. 4619-4625.

https://doi.org/10.1063/1.1602946

This Article is brought to you for free and open access by the Science and Technology Faculty Articles and Research at Chapman University Digital Commons. It has been accepted for inclusion in Mathematics, Physics, and Computer Science Faculty Articles and Research by an authorized administrator of Chapman University Digital Commons. For more information, please contact laughtin@chapman.edu. 


\section{Refrigerator with Phonon Filters: An Application of the Phonon Deficit Effect in Superconducting Tunnel Junctions}

\section{Comments}

This article was originally published in Journal of Applied Physics, volume 94, issue 7, in 2003.

https://doi.org/10.1063/1.1602946

\section{Copyright}

American Institute of Physics 
AIP Appilied Physics

Refrigerator with phonon filters: An application of the phonon deficit effect in superconducting tunnel junctions

G. G. Melkonyan, H. Kröger, and A. M. Gulian

Citation: J. Appl. Phys. 94, 4619 (2003); doi: 10.1063/1.1602946

View online: http://dx.doi.org/10.1063/1.1602946

View Table of Contents: http://jap.aip.org/resource/1/JAPIAU/v94/i7

Published by the AIP Publishing LLC.

Additional information on J. Appl. Phys.

Journal Homepage: http://jap.aip.org/

Journal Information: http://jap.aip.org/about/about_the_journal

Top downloads: http://jap.aip.org/features/most_downloaded

Information for Authors: http://jap.aip.org/authors

\section{ADVERTISEMENT}

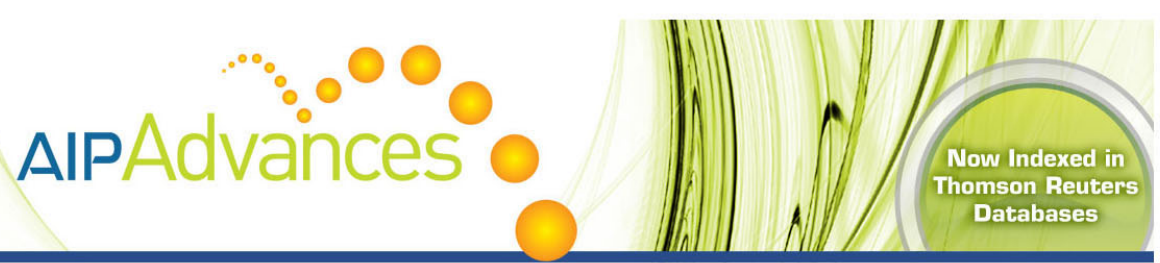

\section{Explore AIP's open access journal: •Rapid publication \\ - Article-level metrics \\ - Post-publication rating and commenting}




\title{
Refrigerator with phonon filters: An application of the phonon deficit effect in superconducting tunnel junctions
}

\author{
G. G. Melkonyan and H. Kröger \\ Département de Physique, Université Laval, Québec, Québec G1K 7P4, Canada
}

A. M. Gulian

Physics Art Frontiers, Naval Research Laboratory, Washington, D.C. 20375

(Received 2 May 2003; accepted 30 June 2003)

\begin{abstract}
Nonequilibrium phenomena in thin solid films can result in cooling effects. These types of effects were predicted theoretically a while ago, and only recently were demonstrated experimentally in superconductor-insulator-normal metal (SIN) tunnel junctions. Since then, there is a growing interest in tunneling effects for the purpose to develop on-chip refrigerators. Thin film devices have the advantage of being extremely compact, operate in a continuous mode, dissipate little power, and can easily be integrated in cryogenic detectors. Currently these refrigerators can generate cooling in the order of $100 \mathrm{mK}$ in an environment of $0.3-0.5 \mathrm{~K}$. There are reasons to expect that this performance can be enhanced but a fundamental investigation of underlying principles is required. One of the outcomes of this type of analysis is the phonon deficit effect. In this article we investigate the phonon deficit effect in thin film superconductor-insulator-superconductor and SIN tunnel junctions. Depending on circumstances, the phonon absorption spectra of such tunnel junctions have spectral windows of phonon emission and/or absorption. We demonstrate that the phonon deficit mechanism can stand for the experimental results with SIN tunnel junctions. In addition, application of the theory of this effect allows us to propose using phonon filters to select the phonon absorption windows and thus to enhance the cooling effect. We discuss a particular superlattice design of corresponding phonon filters. (C) 2003 American Institute of Physics. [DOI: 10.1063/1.1602946]
\end{abstract}

\section{INTRODUCTION}

The possibility of effective cooling opened horizons for both science and technology. One of the most challenging tasks in this area is related to the all-solid state microrefrigerators (SSMR). Those SSMRs have many advantages over conventional cooling devices. The SSMRs are based on the motion of electrons rather than atoms and molecules. They have a long life-time, they are compact and simple to apply.

One of the approaches to microrefrigerators is based on thermoelectricity. The flow of an electric current through a semiconductor-semiconductor contact is accompanied by a transfer of heat (the Peltier effect) from one semiconductor to the other. Microrefrigerators based on the Peltier effect are working at temperatures above $T_{\min } \approx 150 \mathrm{~K}$. The failure of these devices to work below this temperature is due to the fact that the heat conductivity of the crystalline lattice becomes essentially larger by reducing the thermoelectric figure of merit $Z=S^{2} \sigma / k$ (here $S$ is the Seebeck coefficient, $\sigma$ and $k$ are the electric and heat conductivities, respectively) below the efficiency level required for cooling (parasitic heat inflow becomes higher than the cooling rate). For this reason SSMRs operating below $T_{\min }$ are a scientific and technical challenge.

Some hopes were raised by the investigation of electron kinetics in superconductor-insulator-normal metal (SIN) or superconductor-insulator-superconductor (SIS) tunnel junctions. ${ }^{1,2}$ Experiments on refrigeration are usually performed on SIN tunnel junctions. The mechanism called "cooling by extraction" is recognized as the underlying prin- ciple of this type of refrigerators. At temperatures much below the critical temperature $T_{c}$ of the superconducting electrode $(S)$ there are few thermally excited quasiparticles left above the superconducting gap $\Delta$, such that tunneling from $S$ to the normal metal electrode $(N)$ can be neglected. Meanwhile, in the normal metal electrode $N$ there are enough electron-like and hole-like thermal excitations near the Fermi energy $E_{F}$ which can tunnel to $S$ and effectively modify the electron distribution in $N$. The rate of electronic heat exchange with the lattice is proportional to $T_{e}^{5}-T_{\mathrm{ph}}^{5}$, where $T_{e}$ is electronic temperature and $T_{\mathrm{ph}}$ is the temperature of phonons. The cooling process is a balance between phonon heat flow, quasiparticle extraction, and Joule heating. The value of the applied potential $V$ on the tunnel junction determines if cooling or heating occurs depending on which process is dominant in the heat transfer balance.

It seems worthwhile to consider SIN and SIS tunnel junctions in more detail. In this article, we will demonstrate a useful theoretical alternative to understand the cooling mechanism in SIN and SIS tunnel junctions-based on the concept of the phonon deficit effect (PDE). The phonon deficit effect was initially predicted ${ }^{3,4}$ to occur when superconductivity is enhanced in high frequency electromagnetic fields (UHF). ${ }^{5}$ Later the PDE was recognized to exist in more general cases. ${ }^{6,7}$ It is related to the violation of detailed balance of kinetic processes in superconductors in the presence of an external field. If a superconducting film is immersed into a heatbath and the electron system goes over to an ex- 


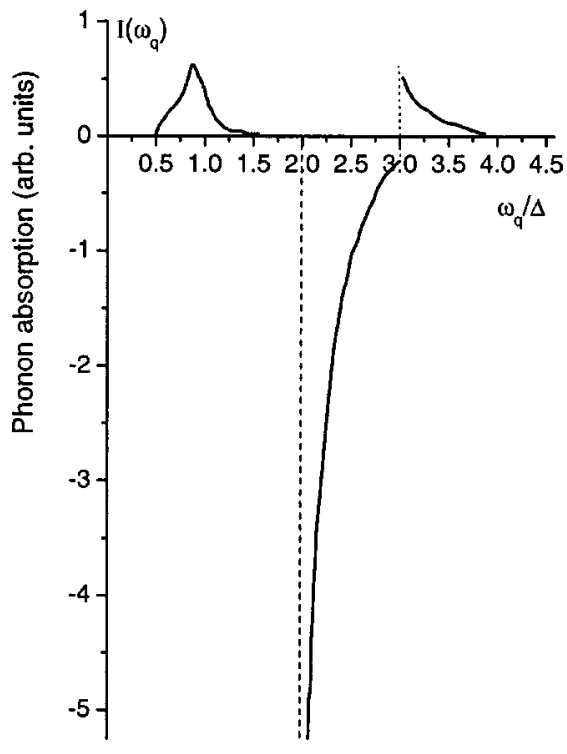

FIG. 1. Spectral dependence of the phonon flux (in terms of energy per unit time) radiated from a thin superconducting film (with smaller gap $\Delta^{S}$ ) in an SIS' tunnel junction (taken from Ref. 6).

cited nonequilibrium state due to an external supply of energy, then under appropriate conditions the superconducting film absorbs phonons from the heatbath rather than emitting them.

The situation is essentially similar in the case of thin tunnel junctions of superconductors. Phonon emission of a symmetric SIS tunnel junction has been considered in detail in Ref. 7 and an asymmetric junction has been considered in Refs. 6, 8, and 9. In the subthreshold tunneling regime ( $V$ $<2 \Delta / e$, where $\Delta$ denotes the gap) the resulting phonon flux is shown in Fig. 1. One observes the phonon emission in the frequency windows $0<\omega_{q}<2 \Delta / \hbar$ and $\omega_{q}>3 \Delta / \hbar$. In the same time there is the phonon absorption in the frequency window $2 \Delta / \hbar<\omega_{q}<3 \Delta / \hbar$. The analogy between this case and the superconductor in the UHF field (see Ref. 6, Fig. 6.2) strikingly demonstrates the universality of the mechanism of the phonon deficit effect.

As we will demonstrate in this article, studying the behavior of SIN tunnel junction on the grounds of the phonon deficit effect allows one not only to reproduce all features of the above mentioned "cooling by extraction," but also opens avenues for efficient refrigeration. The outline of this article is as follows. In Sec. II, we describe the phonon heatbath model and the decoupling of electrons from phonons. In Sec. III, we solve the kinetic equations for the SIN tunnel junction. We calculate the net phonon flux to the SIN tunnel junction when electron and phonon systems are maintained at different temperatures. We show that the rule for heat exchange $P \propto T_{e}^{5}-T_{\mathrm{ph}}^{5}$ follows from the PDE in SIN tunnel junctions. It becomes clear at this point that the PDE is physically equivalent to cooling by extraction. The analysis of cooling in terms of the PDE leads directly to the concept of the phonon filters. We present the results of numerical simulations for phonon filters, which can be utilized to enhance the refrigeration effect. A possible application of phonon filters in accordance with the calculated phonon absorp- tion spectra is discussed in Sec. IV. Conclusions are given in Sec. V.

\section{PHONON HEATBATH MODEL AND DECOUPLING OF ELECTRONS FROM PHONONS}

Experimentally, junction electrodes are thin films placed on a substrate. These thin films should be considered as a system of two interacting subsystems: electrons and phonons. Nonequilibrium dynamics of phonons and electrons in superconductors is described by a coupled system of kinetic equations. ${ }^{6}$ In the simplest case, when the lattice phonons play the role of a thermostat, the kinetic equations for electron (hole) excitations and phonons in a metal can be written in the following form: ${ }^{6}$

$$
\begin{aligned}
& \frac{\partial n_{ \pm \epsilon}}{\partial t}=Q^{f}\left(n_{ \pm \epsilon}\right)+I^{\mathrm{el}-\mathrm{ph}}\left(n_{ \pm \epsilon}, N_{\omega_{q}}^{i}\right), \\
& \frac{\partial N_{\omega_{q}}^{i}}{\partial t}=L\left(N_{\omega_{q}}^{i}\right)+I^{\mathrm{ph}-\mathrm{el}}\left(N_{\omega_{q}}^{i}, n_{ \pm \epsilon}\right) .
\end{aligned}
$$

Here, $n_{ \pm \epsilon}$ denotes the distribution functions of nonequilibrium electron-like $\left(n_{\epsilon}\right)$, respectively, hole-like $\left(n_{-\epsilon}\right)$ excitations in the film. $N_{\omega}^{i}$ represents the distribution function of internal phonons, $I^{\mathrm{el}-} \mathrm{ph}\left(n_{ \pm \epsilon}, N_{\omega_{q}}^{i}\right)$ stands for the electronphonon collision integral, and $I^{\mathrm{ph}-\mathrm{el}}\left(N_{\omega_{q}}^{i}, n_{ \pm \epsilon}\right)$ denotes the phonon-electron collision integral. $Q^{f}\left(n_{ \pm \epsilon}\right)$ is an external source of nonequilibrium quasiparticles and $f$ represents external fields. $L\left(N_{\omega_{q}}^{i}\right)$ is an operator describing the interaction of phonons with an external heatbath.

An important problem in a dynamical theory of metals is the study of nonequilibrium stationary states, i.e., when $\partial n_{ \pm \epsilon} / \partial t=0, \partial N_{\omega_{q}}^{i} / \partial t=0$. For thin films in an external field the dynamical problem can be simplified by considering the phonon heatbath model" 6,10 and also the "geometricacoustical" approximation of phonon propagation. In this approximation the distribution function of internal phonons is given by $N_{\omega_{q}}^{i}=N_{\omega_{q}}^{0}$, where $N_{\omega_{q}}^{0}$ is the distribution function of phonons at thermodynamic equilibrium. The distribution of electrons and holes corresponds to a nonequilibrium state. In a steady state, using the phonon heatbath model, the following system of equations holds:

$$
\begin{aligned}
& -Q^{f}\left(n_{ \pm \epsilon}\right)=I^{\mathrm{el}-\mathrm{ph}}\left(n_{ \pm \epsilon}, N_{\omega_{q}}^{0}\right), \\
& -L\left(N_{\omega_{q}}^{0}\right)=I^{\mathrm{ph}-\mathrm{el}}\left(N_{\omega_{q}}^{0}, n_{ \pm \epsilon}\right) .
\end{aligned}
$$

These equations are uncoupled. Equation (3) can be used to obtain stationary steady state solutions for the distribution functions of nonequilibrium excitations (electron and hole branches). Equation (4) then describes the phonon exchange (phonon flux) between the thin film and its environment. ${ }^{6}$

\section{SIN TUNNEL JUNCTION-KINETIC EQUATIONS IN THE STEADY STATE}

We consider now a massive superconductor (injector $S^{\prime}$ ), having a superconducting gap $\Delta^{\prime}$, joined with a thermostat held at temperature $T$. A thin film (superconductor or normal metal) having a thickness $d \sim \xi_{0}$ is attached to the 
massive superconductor by means of a thin oxide layer. It has the same temperature (see Fig. 4). In the dissipative steady state, nonequilibrium electron $\left(n_{\epsilon}\right)$ and hole $\left(n_{-\epsilon}\right)$ excitations in the thin film ( $S$ or $N$ ) are described by the following kinetic equation [see Eqs. (1) and (3)]:

$$
u_{\epsilon} \frac{\partial n_{ \pm \epsilon}}{\partial t}=Q\left(n_{ \pm \epsilon}\right)+I^{\mathrm{el}-\mathrm{ph}}\left(n_{ \pm \epsilon}, N_{\omega}^{0}\right)=0 .
$$

Here, $u_{\epsilon}=|\epsilon| \theta\left(\epsilon^{2}-\Delta^{2}\right) / \sqrt{\epsilon^{2}-\Delta^{2}}$ denotes the BCS density of states, $\epsilon$ is the quasiparticle energy, and $Q\left(n_{ \pm \epsilon}\right)$ $\equiv Q^{f}\left(n_{ \pm \epsilon}\right)$ describes the injector. In a particular geometry the external field $f$ represents the distribution function of quasiparticles in the electrode denoted by $S^{\prime}$. The explicit form of the electron-phonon collision integral $I^{\mathrm{el}-\mathrm{ph}}\left(n_{\epsilon}, N_{\omega_{q}}^{0}\right)$ and $Q\left(n_{ \pm \epsilon}\right)$ are given in Refs. 4 and 6. The dissipative steady state solutions of Eq. (5) give the electron and the hole distribution functions in the thin film electrode. The corresponding phonon fluxes emitted to (or absorbed from) its environment in a frequency interval $d \omega_{q}$ centered at frequency $\omega_{q}$ can be obtained from Eq. (4).

The number of phonons absorbed per time unit in the volume $\Omega$ at frequency $\omega_{q}$ in the spectral interval $\mathrm{d} \omega_{q}$ is given by

$$
\mathrm{d} \dot{N}_{\omega_{q}}=-L\left(N_{\omega_{q}}^{0}\right) \rho_{0}\left(\omega_{q}\right) \mathrm{d} \omega_{q}=\rho_{0}\left(\omega_{q}\right) I^{\mathrm{ph}-\mathrm{el}}\left(N_{\omega_{q}}^{0}\right) \mathrm{d} \omega_{q} .
$$

Here, $\rho_{0}\left(\omega_{q}\right)=\Omega \omega_{q}^{2} /\left(2 \pi^{2} u^{3}\right), u$ is the velocity of sound, and $\dot{X} \equiv \partial X / \partial t$. The absorbed power per volume $\Omega$ at frequency $\omega_{q}$ in the spectral interval $\mathrm{d} \omega_{q}$ is given by

$$
\frac{\mathrm{d} P}{\mathrm{~d} \omega_{q}}=\hbar \omega_{q} \rho_{0}\left(\omega_{q}\right) I^{\mathrm{ph}-\mathrm{el}}\left(N_{\omega_{q}}^{0}\right) .
$$

Introducing the operator $I\left(N_{\omega_{q}}^{0}\right)$ via

$$
I\left(N_{\omega_{q}}^{0}\right)=\frac{16 \pi \epsilon_{F}}{\lambda \omega_{D}} \omega_{q}^{3} I^{\mathrm{ph}-\mathrm{el}}\left(N_{\omega_{q}}^{0}\right),
$$

we express the absorbed power (7) in dimensionless form

$$
\begin{aligned}
& \frac{\mathrm{d} P}{\mathrm{~d} x}=\frac{\Omega \lambda \omega_{D}}{16 \pi \epsilon_{F} u^{3}} \frac{\left(k_{B} T_{c^{\prime}}\right)^{5}}{\hbar^{3}} I\left(N_{x}^{0}\right)=P_{0} I\left(N_{x}^{0}\right), \\
& P_{0}=\frac{\Omega \lambda \omega_{D}}{16 \pi \epsilon_{F} u^{3}} \frac{\left(k_{B} T_{c^{\prime}}\right)^{5}}{\hbar^{3}},
\end{aligned}
$$

where we abbreviate $x=\hbar \omega_{q} /\left(k_{B} T_{c^{\prime}}\right)$ and $T_{c^{\prime}}$ denotes the critical temperature of the injector.

In the following we use the units $k_{B}=\hbar=e=1$ if not stated otherwise. Also, recall that the primed variables correspond to the injector material.

\section{A. Numerical results and analysis}

To simplify further the analysis, we need to analyze properties of Eq. (5). The electron-phonon collision integral $I^{\mathrm{el}-\mathrm{ph}}$ is proportional to the parameter $\gamma \propto T^{3} / \omega_{D}^{2}$ (damping) which characterizes the strength of the relaxation processes. The parameter $\nu$ describes the intensity of the particle and hole injection $Q\left(n_{ \pm \epsilon}\right)$. The typical values of $\nu$ are small compared to $\gamma$. When $\nu / \gamma$ tends to zero, the particle and hole
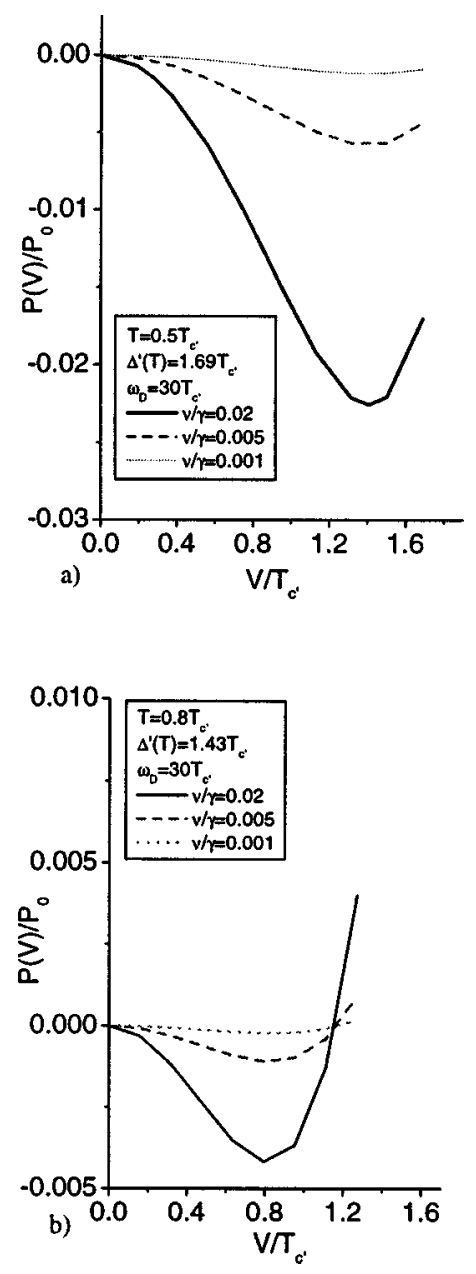

FIG. 2. Absorbed power $P(V)$, in units of $P_{0}$, by the normal metal junction of the thin film as a function of applied potential for different values of $\nu / \gamma$. $\Delta^{\prime}(T)$ and $T_{c^{\prime}}$ are, respectively, the superconducting gap and the critical temperature of the injector, $\omega_{D}$ is the Debye frequency of the normal metal electrode of the junction, and $V$ is the applied potential across the tunnel junction. (a) $T / T_{c^{\prime}}=0.5$, (b) $T / T_{c^{\prime}}=0.8$. The lines are a guide to the eye.

distribution functions go over to thermal equilibrium distribution functions and both, $I^{\mathrm{el}-\mathrm{ph}}\left(n_{ \pm \epsilon}, N_{\omega_{q}}^{0}\right)$ and $I^{\mathrm{ph}-\mathrm{el}}\left(N_{\omega_{q}}^{0}, n_{ \pm \epsilon}\right)$ vanish. At finite values of $\nu / \gamma$ the particle and hole distribution functions differ from the thermal equilibrium functions and thus phonon fluxes arise. As observed in Refs. 4, 8, and 9 both negative and positive phonon fluxes occur between the thin film and the thermal reservoir. The net phonon flux integrated over all frequencies can be either negative or positive.

Figure 2 shows the power $P(V) / P(0)$ absorbed by the normal metal thin film electrode. It is shown as a function of the potential applied across the $S^{\prime} I N$ tunnel junction for different values of the parameter $\nu / \gamma$. All curves have a minimum (maximal absorption) at $V<\Delta^{\prime}(T)$. When the potential difference $V$ across the tunnel junction approaches the superconducting gap $\Delta^{\prime}(T)$, the phonon absorption decreases. The absorbed power increases with the increasing values of $\nu / \gamma$. The situation changes dramatically in the high temperature regime $T / T_{c^{\prime}}>0.5$. When the value of the potential applied across the tunnel junction approaches the superconducting gap $\Delta^{\prime}$, then the $S^{\prime}$ IN tunnel junction starts to emit 

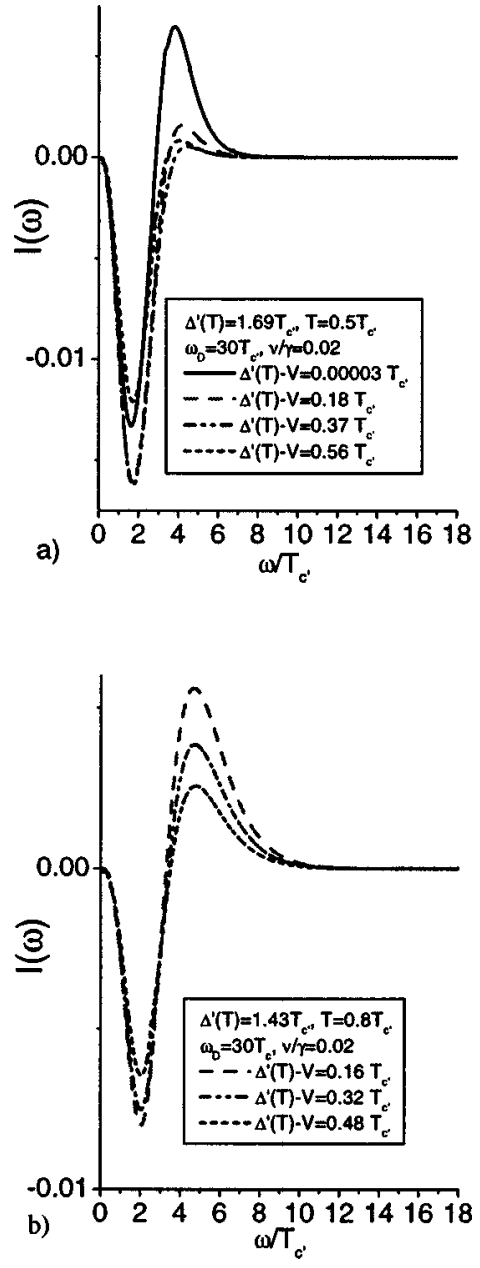

FIG. 3. Spectral absorption (negative part) and emission (positive part) of phonons near the maximal phonon absorption potential of the junction. $\Delta^{\prime}(T)$ and $T_{c^{\prime}}$ are, respectively, the superconducting gap and the critical temperature of the injector, $\omega_{D}$ is the Debye frequency of the normal metal electrode of the junction, and $V$ is the applied potential across the tunnel junction. (a) $T / T_{c^{\prime}}=0.5$, (b) $T / T_{c^{\prime}}=0.8$.

phonons to the environment. This is shown in Fig. 2(b). In Fig. 3 we present the dynamics of phonon flux in the vicinity of the minima of Figs. 2(a) and 2(b). As expected from Fig. 2(a), the phonon absorption spectrum is spread out over the whole frequency range of acoustic phonons at the temperature $T / T_{c^{\prime}}=0.5$ and $\Delta^{\prime}-V>0.18$. At temperature $T / T_{c}^{\prime}$ $=0.8$, there is both phonon emission and phonon absorption at high and low frequency regions, respectively. The phonon emission spectrum of a SIN tunnel junction is different from the $S^{\prime}$ IS case (see Fig. 1). The SIS tunnel junction has two emission and one absorption frequency regions. In SIN tunnel junctions the low frequency emission region is absent. Also, as temperature decreases the phonon emission decreases faster than the absorption.

\section{B. PDE in the SIN tunnel junctions: "equilibrium" limit}

An analytic expression for the power absorbed by the SIN tunnel junction when electron and phonon subsystems are maintained at temperature $T_{e}$ and $T_{\mathrm{ph}}$, respectively, can be obtained by integrating expression (8). The heatflow from the phonon subsystem to the electron subsystem maintained at different temperatures is given then by

$$
P=\alpha\left[\left(\frac{T_{e}}{T_{c^{\prime}}}\right)^{5}-\left(\frac{T_{\mathrm{ph}}}{T_{c^{\prime}}}\right)^{5}\right],
$$

where $\alpha=4 P_{0} \Gamma(5) \zeta(5), \zeta(x)$ denotes Riemann's zeta function and $\Gamma(n)$ is the gamma function. To derive expression (9) one can start from the general expression for the phonon flux given by [compare with the formula (7), $\hbar=1$, $k_{B}=1$ ]

$$
P=\int_{0}^{\omega_{D}} \omega_{q} \rho_{0}\left(\omega_{q}\right) I^{\mathrm{ph}-\mathrm{el}}\left(N_{\omega_{q}}^{0}\right) \mathrm{d} \omega_{q} .
$$

Here, $I^{\mathrm{ph}-\mathrm{el}}\left(N_{\omega_{q}}^{0}\right)$ is the phonon-electron collision integral and it is given by

$$
\begin{aligned}
I^{\mathrm{ph}-\mathrm{el}}\left(N_{\omega_{q}}\right)= & \frac{\pi \lambda \omega_{D}}{8 \epsilon_{F}} \int_{0}^{\infty} \mathrm{d} \epsilon \int_{0}^{\infty} \mathrm{d} \epsilon^{\prime}\left\{\mathcal{S}_{1} \delta\left(\epsilon+\epsilon^{\prime}-\omega_{q}\right)\right. \\
& \left.+2 \mathcal{S}_{2} \delta\left(\epsilon-\epsilon^{\prime}-\omega_{q}\right)\right\},
\end{aligned}
$$

where $\mathcal{S}_{1}$ and $\mathcal{S}_{2}$ are elementary collision processes. When the distribution function of electrons and holes is Fermi function then $n_{\epsilon}=n_{-\epsilon}$. In this case $\mathcal{S}_{1}$ and $\mathcal{S}_{2}$ can be written as $\mathcal{S}_{1}\left(\epsilon, \epsilon^{\prime}, \omega_{q}\right)=4\left[\left(N_{\omega_{q}}+1\right) n_{\epsilon} n_{\epsilon^{\prime}}-N_{\omega_{q}}\left(1-n_{\epsilon}\right)\left(1-n_{\epsilon^{\prime}}\right)\right]$,

$\mathcal{S}_{2}\left(\epsilon, \epsilon^{\prime}, \omega_{q}\right)=4\left[\left(N_{\omega_{q}}+1\right) n_{\epsilon}\left(1-n_{\epsilon^{\prime}}\right)-N_{\omega_{q}}\left(1-n_{\epsilon}\right) n_{\epsilon^{\prime}}\right]$.

After substitutions of expressions (12), (13), and (11) into expression (10), the integration by delta function gives

$$
\begin{aligned}
P= & \frac{4 \pi \lambda \omega D}{8 \epsilon_{F}} \int_{0}^{\omega_{D}} \mathrm{~d} \omega_{q} \omega_{q} \rho_{0}\left(\omega_{q}\right)\left[\int_{0}^{\omega_{q}} \mathcal{S}_{1}\left(\epsilon, \omega_{q}-\epsilon, \omega_{q}\right) \mathrm{d} \epsilon\right. \\
& \left.+\int_{\omega_{q}}^{\omega_{D}+\omega_{q}} 2 \mathcal{S}_{2}\left(\epsilon, \epsilon-\omega_{q}, \omega_{q}\right) \mathrm{d} \epsilon\right] .
\end{aligned}
$$

Finally evaluating these integrals by noting that $T \ll \omega_{D}$ and using the integral representation for Riemann zeta function $\zeta(n+1) \Gamma(n+1)=\int_{0}^{\infty} x^{n} /[\exp (x)-1] d x$ one obtains

$$
P=4 \frac{\Omega}{16 \pi} \frac{\lambda \omega_{D}}{\epsilon_{F} u^{3}} \Gamma(5) \zeta(5)\left(T_{e}^{5}-T_{\mathrm{ph}}^{5}\right) .
$$

A comparison of Eq. (9) with Eq. (19) of Ref. 11 shows that $P \propto T_{e}^{5}-T_{\mathrm{ph}}^{5}$ is compatible with the PDE in SIN tunnel junctions (see Refs. 12 and 13). Obviously, in the usual approach $^{1,2,13,14}$ details like absorption at low frequency region and emission at high frequency region (see Fig. 3) are inaccessible. However, the details of phonon absorption spectra are important and may open new possibilities for cooling by SIN tunnel junctions as discussed in the next section.

\section{MICROREFRIGERATION AND THE PHONON DEFICIT EFFECT}

Why is it important to know the spectral dependence of the phonon fluxes? How can the phonon-deficit-effect influ- 


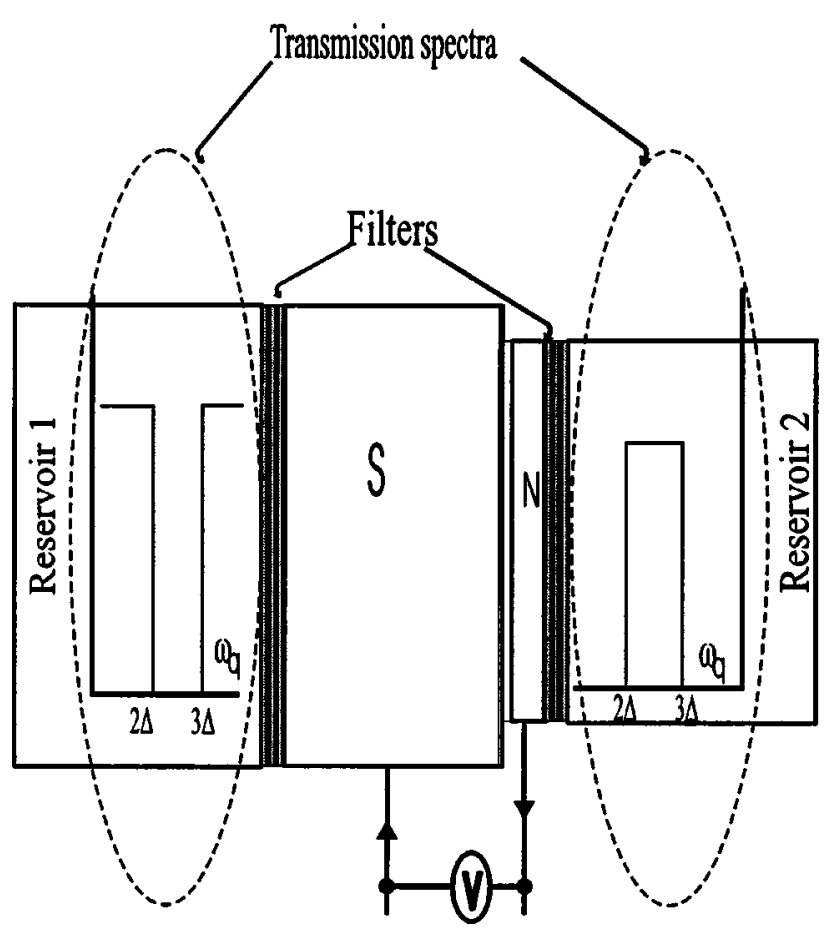

FIG. 4. Layout of the microrefrigerator with the improved properties. Insets show suggested spectral characteristics of superlattice filters.

ence the microrefrigerator performance? The answers to these questions can be found in considering spectralselective phonon filters.

To clarify this point, we should calculate the energy, which the nonequilibrium phonons yield to the heatbath. This quantity is equal to the integral of the spectral function plotted in Fig. 1. This integral having a net negative value is likely to be higher in the case of an asymmetric $S^{\prime} I S$ junction $^{8,9}$ and also in asymmetric $\mathrm{S}^{\prime}$ IN junctions. Figure 1 displays the nonequilibrium phonon flux from the $S^{\prime} I S$ tunnel junction, $\Delta_{S} \ll \Delta_{S^{\prime}}$. As follows from Figs. 1, 2, and 3 the net flux integrated over energy is negative. ${ }^{8,9,15}$ To enhance the PDE effect and achieve better cooling, one should separate nonequilibrium negative and positive fluxes across the interface between the superconductor and the attached heat reservoir. Let us consider placing a phonon spectral filter between superconductor $S$ and reservoir 2 (see Fig. 4). Suppose we can construct a filter ${ }^{16,17}$ which is transparent to phonons in a window of frequencies where the flux in Fig. 1 is negative. Such a filter would permit the flow of phonons $\left(\hbar \omega_{q} \approx 2 \Delta\right.$ ) from reservoir 2 to the superconductor $S$. At the same time the filter will absorb phonons below and above that frequency window, including those coming from the heated barrier. Another filter with complementary (i.e., the bandpass) transmission properties may be placed between reservoir 1 and the electrode $S^{\prime}$. It will prevent phonons coming from $S^{\prime}$ (i.e., reservoir 1) from being absorbed in $S$. This will make the absorption from reservoir 2 more efficient. Then the system shown in Fig. 1 will work as a refrigerator, by cooling reservoir 2 . This cooling effect cannot reduce the temperature much below the critical temperature of superconductor $S^{\prime}$ (see Fig. 1). However, there are different classes of superconductors with critical temperatures cover-
TABLE I. Maximal absorbed power $P / P_{0}$ in the SIN tunnel junction at three different temperatures $T=0.27 T_{c^{\prime}}, T=0.5 T_{c^{\prime}}$, and $T=0.8 T_{c^{\prime}}$.

\begin{tabular}{lrccccc}
\hline \hline$T / T_{c^{\prime}}$ & \multicolumn{2}{c}{0.27} & \multicolumn{2}{c}{0.5} & \multicolumn{2}{c}{0.8} \\
\hline$V / T_{c^{\prime}}$ & \multicolumn{2}{c}{1.56} & \multicolumn{2}{c}{1.31} & \multicolumn{2}{c}{0.79} \\
$\nu / \gamma$ & 0.005 & 0.02 & 0.005 & 0.02 & 0.005 & 0.02 \\
$P / P_{0}$ & -0.004 & -0.014 & -0.0052 & -0.0224 & -0.0011 & -0.0042 \\
\hline \hline
\end{tabular}

ing a wide range of temperatures from about $150 \mathrm{~K}$ to very low temperatures. Then, cooling cascades can be organized in such a way to generate substantial cooling. We suggest that such designs of a PDE-based microrefrigerator could be carefully considered for the purpose of practical use. Some design for phonon filters for the SIS tunnel junctions are discussed in the Appendix. Here we will show that the phonon filters will increase the expected cooling effect.

\section{A. Phonon filters and refrigeration}

The proposed PDE refrigerator is suited to refrigerate an object attached to it. The effect of phonon filters can be estimated by the value of $P / P_{0}$ from an experiment carried out by Manninen et al. ${ }^{13}$ where a membrane attached to the SIN tunnel junction was cooled. Our estimate for the net phonon absorption $P / P_{0}$ by Eq. (9) varies from 0 to $10^{-2}$ depending on temperatures $T_{e}$ and $T_{\mathrm{ph}}$. Table I presents our calculated values for the nonequilibrium tunnel junctions (see also Fig. 3). We find qualitative agreement between experiment and calculation for the value of absorbed power.

The phonon filters will increase the absorbed power (see Fig. 3) by cutting off the emission window. Table II presents the absorbed power after placing a low pass filter (see Figs. 3 and 5) between the SIN tunnel junction and an object. Phonon absorption is increased at $T / T_{c}=0.5$. At $T / T_{c}=0.8$ we see that SIN tunnel junction with phonon filter will absorb phonons (the minus sign in Table II means absorption). Figure 7 shows the temperature dependence of the total phonon absorption $P / P_{0}$ for three different cases. The solid line is the phonon absorption by a SIN tunnel junction without phonon filter. The dotted and dashed curves present the filtered phonon absorption for two different low pass filters with transmittance 1 at $\omega<2.95 T_{c}$ and $\omega<3.25 T_{c}$. Thus the phonon absorption is increased in a wide temperature region.

We recall that the refrigeration schema presented above is suited to refrigerate an object attached to it. But as follows from the phonon absorption spectra an analogous design with high pass filters can prevent the phonon absorption by SIN tunnel junction and thus reduce the heating of electrons by phonons (see Figs. 3 and 5).

TABLE II. Absorbed power $P / P_{0}$ in the SIN tunnel junction at two different temperatures $T=0.5 T_{c^{\prime}}$ and $T=0.8 T_{c^{\prime}}$, without and with phonon filter. The data below correspond to Fig. 3. The ratio $\nu / \gamma=0.02$.

\begin{tabular}{lccccc}
\hline \hline$T / T_{c^{\prime}}$ & \multicolumn{3}{c}{0.5} & \multicolumn{2}{c}{0.8} \\
\hline$V / T_{c^{\prime}}$ & & 1.31 & & 1.31 & \\
Effect & total & filtered & total & filtered \\
$P / P_{0}$ & $-2.2410^{-2}$ & $-2.4310^{-2}$ & $1.2410^{-4}$ & $-2.510^{-2}$ \\
\hline \hline
\end{tabular}




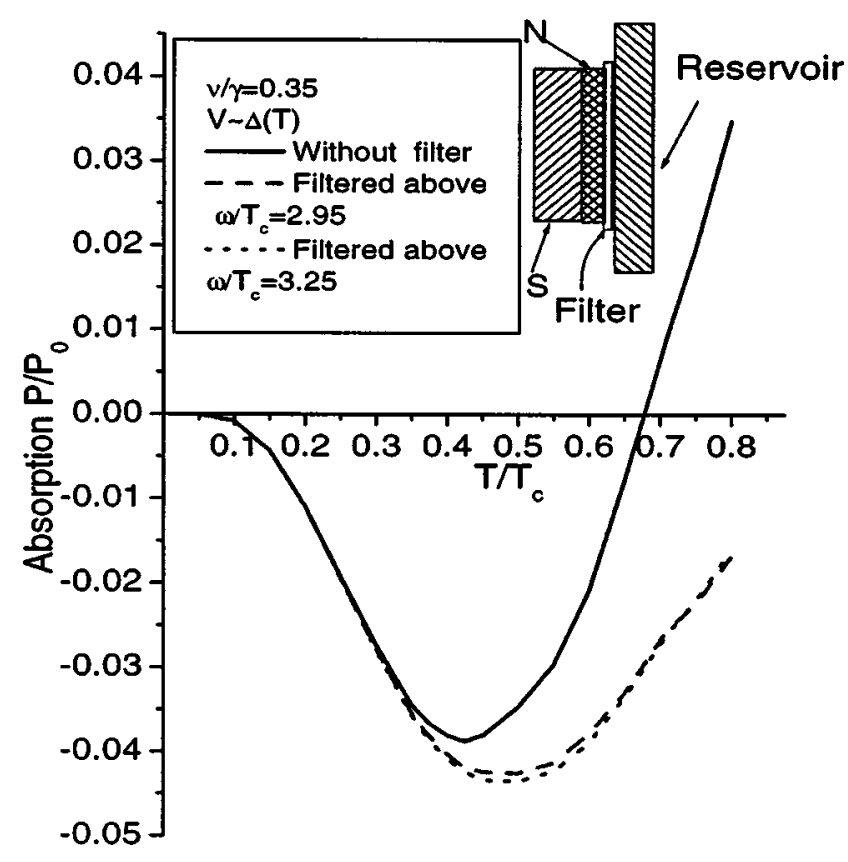

FIG. 5. Phonon flux with low pass filter at $\omega=2.95 T_{c}$ and $\omega=3.25 T_{c}$.

\section{CONCLUSIONS}

The SSMR performance depends on various external and internal parameters. The PDE-based approach presented here and the usual approach ${ }^{11-13}$ give the similar expression [Eq. (9)] for the net cooling in case of SIN tunnel junction. Thus one can conclude that the cooling of a membrane in the experiment by Manninen et al. ${ }^{13}$ can be treated as a direct implication of the phonon deficit effect. The PDE approach offers explicit spectral description for participating phonon fluxes. The benefit of the description of nonequilibrium junctions in terms of the PDE is in providing receipts to over-

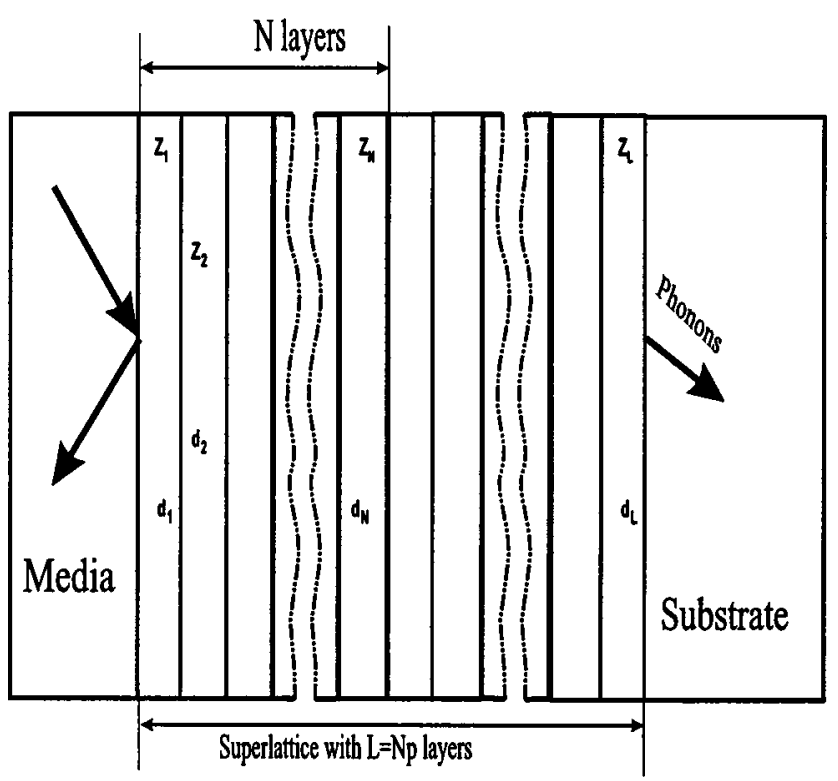

FIG. 6. Bragg multilayer between two media.
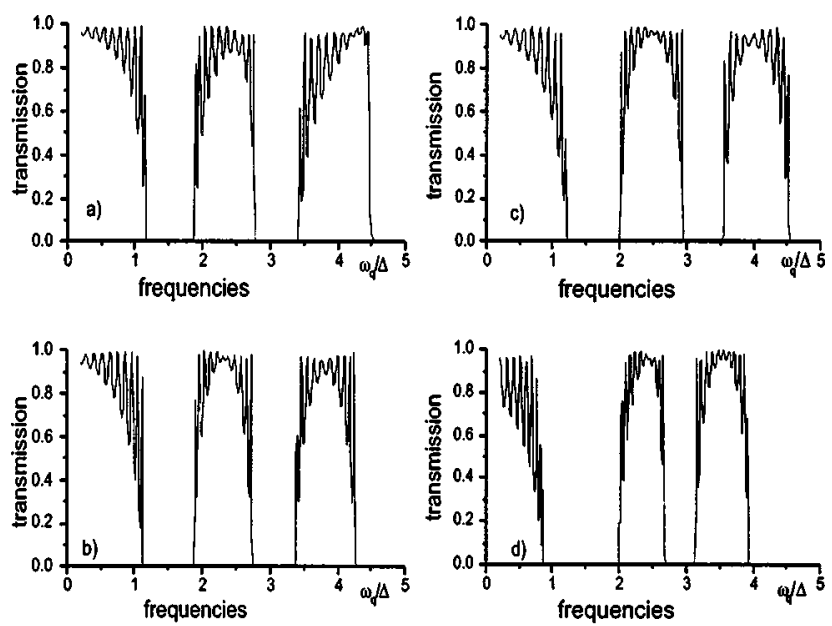

FIG. 7. Band pass properties of designed filters (for the parameters see Table III).

come unwanted heating channels and shows new details which have been omitted in a simplified "cooling by extraction" description.

Application of the "phonon deficit effect" to the SIN tunnel junctions reveals that in contrast to the SIS tunnel junctions there is a temperature region where the SIN tunnel junctions essentially absorb low frequency phonons. As temperature is increased, the SIN tunnel junctions also emit phonons to the environment. Depending on the value of the ratio $\nu / \gamma$ there is net phonon emission or absorption. The temperature dependence of the net phonon absorption is consistent with experimental observations: ${ }^{1,2,13}$ there is heating at high and low temperatures and refrigeration at intermediate temperatures.

To enhance the cooling power of the $\operatorname{SIS}(N)$ and tunnel junctions and overcome the phonon heating at high temperatures, phonon filters can be effective. We performed calculations for the microrefrigerator phonon filters. Those filters have superlattice design and can be obtained using contemporary nanotechnology methods. They can enhance the cooling power of SIS and SIN tunnel junctions.

\section{ACKNOWLEDGMENTS}

H.K. has been supported by NSERC Canada. G.M. is grateful for support by the "Program Québécois de Bourses d'Excellance" of the Governement of Québec.

TABLE III. Parameters of the presented filters (see Fig. 7). The impedance $(Z)$ and the size of the layers $(d)$ are given in units of $10^{5} \mathrm{~g} /\left(\mathrm{s} \mathrm{cm}^{2}\right)$ and in angstroms $(\AA)$, respectively.

\begin{tabular}{lllllllllllll}
\hline \hline & $Z_{1}$ & $Z_{2}$ & $Z_{3}$ & $Z_{4}$ & $Z_{5}$ & $d_{1}$ & $d_{2}$ & $d_{3}$ & $d_{4}$ & $d_{5}$ & $\mathrm{~N}$ & $\mathrm{p}$ \\
\hline $\mathrm{a}$ & 2.5 & 1.8 & 1.4 & 0.9 & 1.8 & 50 & 50 & 50 & 50 & 30 & 5 & 11 \\
$\mathrm{~b}$ & 3 & 1.8 & 1.3 & 0.9 & 1 & 50 & 50 & 50 & 50 & 30 & 5 & 11 \\
$\mathrm{c}$ & 3 & 1.8 & 1.3 & 0.9 & 1 & 40 & 45 & 50 & 50 & 30 & 5 & 11 \\
$\mathrm{~d}$ & 3 & 1.8 & 1.3 & 0.9 & 1 & 40 & 40 & 40 & 45 & 30 & 5 & 11 \\
\hline
\end{tabular}




\section{APPENDIX: FILTERING PHONON FLUXES}

From the analysis in the Sec. IV it follows that the design of appropriate phonon filters is of primary importance. Using the analogy between reflection of a plane electromagnetic wave at the interface between two optical media with different refractive indexes $n_{1}$ and $n_{2}$ and the acoustic wave reflecting at the boundary of two elastic media with different acoustic impedance $Z_{1}$ and $Z_{2}$, Narayanamurti et al..$^{18}$ and Koblinger et al. ${ }^{19}$ have developed appropriate phonon filters for phonon spectrometry.

Here we consider the simplest case of a phonon filter ${ }^{9,17}$ for the case when acoustic phonons propagate through a superlattice perpendicular to interfaces. In Fig. 6, the superlattice system is shown. The system consists of a sequence of different layers: material 1 with thickness $d_{1}$ and impedance $Z_{1}$, material 2 with thickness $d_{2}$ and impedance $Z_{2}$, etc. If one repeats this configuration $p$ times then the superlattice will have a period $D=d_{1}+d_{2}+\cdots+d_{n}$ and $L=N p$ layers. The interfaces are perpendicular to the wave vector of the incident wave. The superlattice is placed between two media with the acoustic impedance $Z_{\text {medium }}$ and $Z_{\text {substrate }}$. Methods of calculation for the transmission and reflection coefficients in a linear stratified medium are described in Refs. 20-23. We have presented the results of our calculation based on theses methods in Fig. 7. One should note that all filters have an oscillatory behavior for the transmission coefficient in the band pass region. This is typical for band pass filters. In Table III we list the impedance and thickness of the superlattices used in our calculations. Figure 7 presents curves for the transmission spectra of the filter between reservoir 2 and superconductor $S$ with different acoustic impedance, layer thickness, and layer number. Comparing with Fig. 1 we observe large phonon fluxes at frequencies about $\omega_{b f q}=\Delta / \hbar$ and $\omega_{b f q}=3 \Delta / \hbar$. The filter shown in Fig. 7(d) may work well for those frequencies. The filter between reservoir 1 and superconductor $S^{\prime}$ is presented in Ref. 17. The phonon filters for the SIN tunnel junctions can be designed by a similar analysis.

${ }^{1}$ M. Leivo, J. Pekola, and D. Averin, Appl. Phys. Lett. 68, 1996 (1996).

${ }^{2}$ P. Fisher, J. N. Ullom, and M. Nahum, Appl. Phys. Lett. 74, 2705 (1999).

${ }^{3}$ A. Gulian and G. Zharkov, Pis'ma Zh. Eksp. Teor. Fiz. 34, 161 (1981); [JETP Lett. 34, 153 (1981)].

${ }^{4}$ A. Gulian and G. Zharkov, Phys. Lett. A 80, 79 (1980).

${ }^{5}$ G. Eliashberg, Pis'ma Zh. Eksp. Teor. Fiz. 11, 186 (1970); [JETP Lett. 11, 114 (1970)].

${ }^{6}$ A. Gulian and G. Zharkov, Electrons and Phonons in Nonequilibrium Superconductors (Kluwer/Plenum, New York, 1999).

${ }^{7}$ A. Gulian and G. Zharkov, Zh. Eksp. Teor. Fiz. 89, 156 (1985); [Sov. Phys. JETP 62, 89 (1985)].

${ }^{8}$ A. Gulian, G. Zharkov, and G. Sergoyan, Kratkie Soobshcheniya po Fizike FIAN 10, 33 (1985).

${ }^{9}$ G. Melkonyan, H. Kröger, and A. Gulian, Physica B 284-288, 2032 (2000).

${ }^{10}$ G. Eliashberg, Zh. Eksp. Teor. Fiz. 61, 1254 (1971); [Sov. Phys. JETP 34, 668 (1972)].

${ }^{11}$ P. Allen, Phys. Rev. Lett. 59, 1460 (1987).

${ }^{12}$ F. Wellstood, C. Urbina, and J. Clarke, Phys. Rev. B 49, 5942 (1994).

${ }^{13}$ A. Manninen, M. Leivo, and J. Pekola, Appl. Phys. Lett. 70, 1885 (1997).

${ }^{14}$ M. Castelano, R. Leoni, G. Torrioli, P. Carelli, and A. Gerardino, Nuovo Cimento D 19, 1417 (1997), paper presented at the VIII Congresso SATT, Como, 1-4 October 1996.

${ }^{15}$ A. Gulian, G. Zharkov, and G. Sergoyan, Sov. Phys. Lebedev. Inst. Rep. 10, 38 (1985).

${ }^{16}$ A. Gulian, Physica B 263-264, 621 (1999).

${ }^{17}$ G. Melkonyan, H. Kröger, and A. Gulian, in International Conference of the Applied Photonic Technologies (ICAPT), Quebec, Proceedings of SPIE, edited by R. Lessard and G. A. Lampuopoulos (SPIE, Québec, 2000), Vol. 4087, p. 525.

${ }^{18}$ V. Narayanamurti, H. Störmer, M. Chin, A. Gossard, and W. Wiegmann, Phys. Rev. Lett. 43, 2012 (1979).

${ }^{19}$ O. Koblinger, J. Mebert, S. D. E. Dittrich, W. Eisenmenger, P. Santos, and L. Ley, Phys. Rev. B 35, 9372 (1987).

${ }^{20}$ D. Pierce, Acoustics: An Introduction to its Physical Principles and Applications (McGraw-Hill, New York, 1981).

${ }^{21}$ P. Richards, Proc. IRE 34, 145 (1946).

${ }^{22}$ S. Shelkunoff, Bell Syst. Tech. J. 17, 17 (1938).

${ }^{23}$ S. L. Chung, Phys. Rev. B 43, 9649 (1991). 